\title{
SOME NEW DISCRETE FRACTIONAL INEQUALITIES AND THEIR APPLICATIONS IN FRACTIONAL DIFFERENCE EQUATIONS
}

\author{
BIN ZHENG
}

Abstract. In this paper, some new Gronwall-Bellman type discrete fractional difference inequalities and fractional sum inequalities are established. Based on the theory of discrete fractional calculus, explicit bounds for unknown functions concerned are presented. These inequalities can be used as a handy tool in the qualitative analysis of solutions of discrete fractional difference equations. As for applications, we apply the presented results to research boundedness, uniqueness, and continuous dependence on the initial value for the solutions of certain initial value problems of fractional difference equations.

Mathematics subject classification (2010): 26D15.

Keywords and phrases: Gronwall-Bellman type inequality, discrete fractional difference inequality, fractional sum inequality, fractional difference equation, qualitative property.

\section{REFERENCES}

[1] B. G. Pachpatte, Inequalities for Differential and Integral Equations, Academic Press, New York, 1998.

[2] L. Z. Li, F. W. Meng, L. L. He, Some generalized integral inequalities and their applications, J. Math. Anal. Appl. 372 (2010) 339-349.

[3] W. N. Li, M. A. HAn, F. W. Meng, Some new delay integral inequalities and their applications, J. Comput. Appl. Math. 180 (2005) 191-200.

[4] W. S. WANG, A class of retarded nonlinear integral inequalities and its application in nonlinear differential-integral equation, J. Inequal. Appl. 2012:154 (2012) 1-10.

[5] W. S. WANG, Some retarded nonlinear integral inequalities and their applications in retarded differential equations, J. Inequal. Appl. 2012:75 (2012) 1-8.

[6] O. Lipovan, Integral inequalities for retarded Volterra equations, J. Math. Anal. Appl. 322 (2006) 349-358.

[7] R. A. C. Ferreira, D. F. M. Torres, Generalized retarded integral inequalities, Appl. Math. Lett. 22 (2009) 876-881.

[8] Y. G. Sun, On retarded integral inequalities and their applications, J. Math. Anal. Appl. 301 (2005) $265-275$.

[9] R. XU AND Y. G. Sun, On retarded integral inequalities in two independent variables and their applications, Appl. Math. Comput. 182 (2006) 1260-1266.

[10] W. S. Cheung, J. L. Ren, Discrete non-linear inequalities and applications to boundary value problems, J. Math. Anal. Appl. 319 (2006) 708-724.

[11] Q. H. MA, Estimates on some power nonlinear Volterra-Fredholm type discrete inequalities and their applications, J. Comput. Appl. Math. 233 (2010) 2170-2180.

[12] W. S. Cheung, Q. H. MA And J. PeČARIĆ, Some discrete nonlinear inequalities and applications to difference equations, Acta Math. Scientia 28 (B) (2008) 417-430.

[13] R. Agarwal, M. Bohner, A. Peterson, Inequalities on time scales: a survey, Math. Inequal. Appl. 4 (4) (2001) 535-557.

[14] W. N. LI, Some delay integral inequalities on time scales, Comput. Math. Appl. 59 (2010) 1929-1936.

[15] S. H. S AKER, Some nonlinear dynamic inequalities on time scales and applications, J. Math. Inequal. 4 (2010) 561-579. 
[16] S. H. SAKER, Some nonlinear dynamic inequalities on time scales, Math. Inequal. Appl. 14 (2011) 633-645.

[17] Q. H. Feng, B. Zheng, Generalized Gronwall-Bellman-type delay dynamic inequalities on time scales and their applications, Appl. Math. Comput. 218 (2012) 7880-7892.

[18] Q. H. Feng, F. W. Meng, B. Zheng, Gronwall-Bellman type nonlinear delay integral inequalities on time scales, J. Math. Anal. Appl. 382 (2011) 772-784.

[19] B. Zheng, Q. H. Feng, F. W. Meng, Y. M. Zhang, Some new Gronwall-Bellman type nonlinear dynamic inequalities containing integration on infinite intervals on time scales, J. Inequal. Appl. 2012:201 (2012) 1-20.

[20] Q. H. Feng, F. W. Meng, Y. M. Zhang, B. Zheng, J. C. Zhou, Some nonlinear delay integral inequalities on time scales arising in the theory of dynamics equations, J. Inequal. Appl. 2011:29 (2011) $1-14$.

[21] H. P. Ye, J. M. GAO, Y. S. Ding, A generalized Gronwall inequality and ins application to a fractional differential equation, J. Math. Anal. Appl. 328 (2007) 1075-1081.

[22] J. Shao, F. W. Meng, Gronwall-Bellman Type Inequalities and Their Applications to Fractional Differential Equations, Abs. Appl. Anal. 2013:217641 (2013) 1-7.

[23] Q. Feng, F. W. Meng, Some new Gronwall-type inequalities arising in the research of fractional differential equations, J. Inequal. Appl. 2013:429 (2013) 1-8.

[24] B. Zheng, Some New Gronwall-Bellman-Type Inequalities Based on the Modified Riemann-Liouville Fractional Derivative, J. Appl. Math. 2013:341706 (2013) 1-8.

[25] J. Shao, New Integral Inequalities with Weakly Singular Kernel for Discontinuous Functions and Their Applications to Impulsive Fractional Differential Systems, J. Appl. Math. 2014:252946 (2014) $1-5$.

[26] G. V. S. R. Deekshitulu, J. J. Mohan, Some new fractional difference inequalities of GronwallBellman type, Math. Sci. 2012, 6:69 (2012) 1-9.

[27] G. V. S. R. Deekshitulu And J. Jagan Mohan, Fractional difference inequalities of GronwallBellman type, acta et commentationes Universitatis Tartuensis de Mathematica, 17 (1) (2013) 1-12.

[28] F. M. Atici, P. W. Eloe, Initial Value Problems In Discrete Fractional Calculus, Proceedings of The Amer. Math. Soc. 137 (3) (2009) 981-989.

[29] F. M. Atici, P. W. Eloe, A Transform Method in Discrete Fractional Calculus, Inter. J. Diff. Equ. 2 (2) (2007) 165-176.

[30] M. Holm, Sum and Difference Compositions in Discrete Fractional Calculus, CUBO A Mathematical Journal 15 (3) (2011) 153-184.

[31] F. C. JiAng, F. W. Meng, Explicit bounds on some new nonlinear integral inequality with delay, J. Comput. Appl. Math. 205 (2007) 479-486. 